Instituto Internacional de Investigación y Desarrollo Tecnológico Educativo INDTEC, C.A.

DOI: https://doi.org/10.29394/scientific.issn.2542-2987.2017.2.6.18.337-351

OAI-PMH: http://www.indteca.com/ojs/index.php/Revista Scientific/oai

\title{
Formación en Cultura Ciudadana Espacio para la Responsabilidad Social de las Organizaciones Empresariales
}

\author{
Autora: Elita Marina Méndez Jiménez \\ Universidad Centroccidental Lisandro Alvarado, UCLA \\ elitamendezj@gmail.com; elitamendez@ucla.edu.ve
}

Lara, Venezuela

\section{Resumen}

En Venezuela convivimos en una época sedienta de paz, de compromiso y convivencia social, de igualdad social, a diario escuchamos decir que hay crisis social, crisis de valores. En esta coyuntura, la educación corresponsablemente impartida es el factor central para reflexionar, inculcar, fortalecer y consolidar en los ciudadanos, valores, formación personal, formación ética y otros temas vinculantes, en suma, cultura ciudadana. Es propósito de este ensayo generar reflexiones en torno a la cultura ciudadana, para ello se mencionan algunos roles, que como acciones gerenciales de corresponsabilidad social, pueden realizar las organizaciones empresariales privadas, para fortalecer el accionar social del individuo en aras de promover los estímulos necesarios para que alcance la condición de buen ciudadano, para quien la idea de vivir en una comunidad próspera y participativa, sea representada en un espacio donde la educación, el buen trato, la igualdad en oportunidades y el respeto a sus semejantes, al hábitat y a la vida en cualquiera de sus expresiones sean la norma.

Palabras clave: comportamiento social; responsabilidad civil; educación ciudadana. 


\title{
Formation in Citizen Culture, Space for the Social Responsibility of Business Organizations
}

\begin{abstract}
In Venezuela, we live together in a time of thirst for peace, commitment and social coexistence, social equality, every day we hear that there is a social crisis, a crisis of values. At this juncture, the education imparted is the central factor in reflecting, inculcating, strengthening and consolidating in the citizens, values, personal formation, ethical training and other binding issues, in short, citizen culture. It is the purpose of this essay to generate reflections around the citizen's culture, for it mentions some roles, that as managerial actions of social co-responsibility, can realize the private business organizations, to strengthen the social action of the individual in order to promote the necessary stimuli to reach the status of a good citizen, for whom the idea of living in a prosperous and participatory community is represented in a space where education, good treatment, equality in opportunities and respect for their fellow human beings, habitat and life in any of its expressions are the norm.
\end{abstract}

Keywords: social behavior; civil responsibility; civic education.

Date Received: 15-05-2017

Date Acceptance: 02-10-2017 


\section{Consideraciones generales.}

La Constitución de la República Bolivariana de Venezuela (1999), establece la tarea de refundar el país, asumir una nueva concepción de ciudadanía y democracia, además de censurar la pasividad, la indiferencia y la falta de solidaridad, tarea en la cual, la educación cobra una especial importancia. Por ello, según Acosta, Páez y Vizcaya (2007), en Venezuela, la educación, en todos sus niveles, debe contribuir al logro del pleno desarrollo de la personalidad de los ciudadanos, para lograr los propósitos constitucionales.

En consonancia con lo anterior, según el Ministerio de Educación y Deportes de la República Bolivariana de Venezuela (2004), y el Ministerio del Poder Popular para la Planificación y el Desarrollo (2009), desde los diferentes niveles del sector educativo se han generado planes, programas y proyectos orientados, al desarrollo de capacidades que permitan ejercer una ciudadanía crítica, participativa y activa. Esta ciudadanía debe estar orientada al desarrollo pleno de la personalidad para el disfrute de una existencia digna, que transcurra con una valoración ética del trabajo y con una conciencia de participación y corresponsabilidad ciudadana. La corresponsabilidad según Anzola (2012), implica una verdadera transformación social de los ciudadanos y por ende de un país y la construcción de un proceso social en donde se conjuguen valores y modos de aprehensión de la realidad política, social, jurídica, en atención a un contexto histórico dado.

La actual problemática social venezolana, representada entre otras, por un incremento de la criminalidad, el irrespeto a las normas y la indiferencia social es un tema de primer orden, que para su solución o disminución requiere, del concurso de todos los sectores, públicos y privados, trabajar de manera intensa y mancomunada en la divulgación, inculcamiento, consolidación y fortalecimiento de valores ciudadanos que permitan a los individuos interrelacionarse de forma armónica, sana y adecuada. 
Es propósito de este ensayo generar reflexiones en torno a la temática mencionada, para ello se mencionan algunos roles, que como acciones gerenciales de responsabilidad social, pueden realizar las organizaciones empresariales privadas, para fortalecer el accionar social del individuo en aras de promover los estímulos necesarios para que alcance mediante la formación en valores, la condición de buen ciudadano, para quien la idea de vivir en una comunidad próspera y participativa, sea representada en un espacio donde la educación, el buen trato, la igualdad en oportunidades y el respeto mutuo sean la norma.

En la actualidad, Venezuela vive un proceso de trasformación social importante, donde varias tendencias convergen en un intento por consolidar teorías diversas sobre el mejor o más indicado modelo de convivencia, integración social, producción y distribución económica, de allí nace la inquietud de abordar un tema que consideramos vital, como lo es la formación de ciudadanos íntegros, con cultura ciudadana y abocados al mejoramiento de su entorno, bien sea familiar, laboral, comunitario, y por qué no, más allá de los límites patrios, haciendo de nuestros ciudadanos un modelo a seguir por otros países.

Se ha hecho común ver el comportamiento displicente e indiferente del habitante para con la ciudad, sin respeto y consideración a sus conciudadanos, a la propiedad pública o privada, dándosele más valor a los medios que a los objetivos. Ante este frecuente comportamiento ciudadano, el cual se observa crecer de forma acelerada y sostenida, queda preguntar. ¿Han sido suficientes los planes y programas de educación en valores desarrollados por los diferentes niveles de la educación? ¿Solo a las familias, instituciones educativas, docentes e instituciones públicas les corresponde desarrollar programas de formación en cultura ciudadana? ¿Qué tipo de responsabilidad tienen las organizaciones empresariales en esta problemática social? ¿Cuáles pudieran ser los aportes de las organizaciones empresariales para alcanzar la 
tan anhelada formación en cultura ciudadana y contribuir al logro de un mejor país?

\section{El ciudadano y ciudadanía. ¿Conceptos de asunción automática?}

Para Horrach (2009), ciudadanía es un concepto de construcción paulatina e histórica, pudiera señalarse que está asociado con la modernidad, sin embargo, en el imaginario social la idea de ciudadanía ha ampliado su ámbito a otros espacios de la cotidianidad social. Así como amplió su radio de acción e interpretación, ha logrado apropiarse de derechos que por la necesidad de la propia definición le pertenecen o le son inherentes, de los que un principio se beneficiaban pequeños grupos, lo cual evidentemente ha cambiado, ahora podemos hablar de igualdad, incluso, de un progreso orientado hacia una redefinición de mayor inclusión que va más allá de desigualdades ideologías, religiosas y de origen geográfico

Con respecto al concepto de ciudadano, Mejías y Henríquez (2012), señalan que es aquel individuo consciente de su rol transformador y emancipador, es comprometido con la igualdad y partícipe de proyectos de construcción colectiva, estas interacciones posibilitan la expansión de las relaciones entre sí y con el Estado, lo cual le facilita el ejercicio de ciudadanía desde lo local propiciando canales de participación.

Por tanto un buen ciudadano es aquel que participa en la construcción de la ciudad justa, en otras palabras, es una persona capaz de lograr cambios o fomentarlos en cooperación con un grupo de similares, los cuales buscan un orden en su entorno que les permita vivir en armonía, necesidad que obliga al establecimiento de leyes, normativas y códigos, formas que pretenden dar carácter formal al comportamiento social ideal y establecer los límites para cualquier miembro de la comunidad en cuestión, con respeto de sus derechos, los cuales junto a las leyes, han sido creados para garantizar la igualdad. 
En esta última definición, están incorporados los conceptos de convivencia, armonía, respeto, solidaridad, colaboración, participación, identidad, características o atributos ciudadanos que deben ser exhibidos, aplicados y mantenidos por quienes se precien de tales.

\section{Necesidad de la Temprana Formación en Cultura Ciudadana.}

Todo movimiento que pretenda impulsar cambios, debe sin duda apoyarse en bases sólidas, que en este caso son representadas en gran parte por la formación de valores, si bien es cierto que la familia, los grupos sociales y los medios de comunicación contribuyen a la formación cívica, esta educación debe ser perfeccionada mediante estudios formales sobre los fundamentos de la organización cívica y social, que a su vez fomenten el desarrollo del entendimiento y aplicación de los deberes y derechos con los cuales se busque aflorar actitudes positivas ante el entorno.

La idea sobre educación ciudadana no es novedosa, con versiones diferentes ha estado en las agendas de las instituciones docentes y en las planificaciones e intenciones gubernamentales, lo cual según Martínez (2010), debe mantenerse pero no limitarse, es decir, llevarla también a otros espacios sociales.

Considerando lo anterior y tomando como principal protagonista la formación temprana de mejores ciudadanos, es primordial resaltar la importancia que tiene el fomentar y desarrollar estos valores en los iniciales niveles de educación, ya que allí están los jóvenes que en poco tiempo ejercerán plenamente sus deberes y derechos ciudadanos dentro de la sociedad, de ahí lo importante de la formación social y ciudadana que deben propiciar las instituciones, públicas o privadas, en tanto actores de la educación venezolana, necesariamente reforzada o iniciada en el seno de la familia. 


\section{Formación en Valores y Cultura Ciudadana. ¿Compromiso Compartido de Responsabilidad Social de las Empresas?}

Para establecer la relación entre RS, empresas y formación en valores ciudadanos, es preciso analizar a las organizaciones empresariales como un universo de corrientes culturales, sociales, éticas entre otras, en las cuales se debería fomentar la educación ciudadana, para afianzar los valores ciudadanos en búsqueda de propiciar un ambiente laboral cómodo para todos y que este comportamiento sea afianzado de tal forma que en algún momento llegue a proyectarse más allá de los limites organizacionales.

Alrededor del concepto de RS, pese a su institucionalización en el medio empresarial, subsisten discrepancias en cuanto a la forma de entenderlo, definirlo y aplicarlo, hay quienes defienden de manera exacerbada la visión de negocios sin importar los resultados e impactos a la sociedad, que los problemas sociales no son asunto del dominio empresarial y que con las inversiones realizadas, los tributos pagados y la creación de puestos laborales ya se da un servicio social y de alguna manera se cumple la RS.

También hay quienes entiende la RS como una manera de revertir a la sociedad parte de sus éxitos y ganancias, porque precisamente es el espacio de sus operaciones y negocios y bajo esta interpretación y según el Fondo Multilateral de Inversiones FOMIN (2011), realizan acciones y prácticas, para dejar de lado enfoques filantrópicos aislados e iniciativas de inversión social desarticuladas, en búsqueda de programas más asociados a la estrategia corporativa, fenómeno que parece estar reforzado por los retos que impone la globalización y el impulso del comercio internacional.

En concreto, podemos decir que una empresa socialmente responsable es aquella que se caracteriza, entre otras prácticas, por tomar en cuenta las necesidades y características de sus relacionados, por direccionar la productividad de sus trabajadores o colaboradores para mejorar su competitividad, el impacto ambiental resultante de su actividad productiva, y la 
comunidad sobre la cual se asienta la empresa, es decir, su entorno social. Debemos destacar que en estas prácticas el concepto de Educación en Cultura Ciudadana no es expresamente mencionado.

Interesados en aportar cambios de valor a la sociedad, apostamos que con una adecuada utilización de medios y herramientas gerenciales, se puede llegar a promover cambios de actitud dentro de las organizaciones, que contribuyan a la creación de conciencia ciudadana, tomando como laboratorio el entorno laboral y organizacional, pretendiendo con esto crear conciencia sobre la importancia de la cooperación ciudadana y el aporte que cada quien debe realizar.

\section{Educación en Cultura Ciudadana. Aseguramiento de Convivencia} Social.

Según Guevara, Zambrano y Evies (2007), vivimos en una época sedienta de paz, compromiso, convivencia social, igualdad social y donde a diario escuchamos decir que hay crisis social, crisis de valores. Ante esta contingencia, la educación es el protagonista central para formar en todos los ámbitos sociales y niveles educativos sobre educación en valores ciudadanos, formación personal, formación ética y otros temas vinculantes, en suma, cultura ciudadana.

Para el Instituto Mexicano para la Competitividad IMCO (2010a), la cultura ciudadana es la demostración consolidada de valores, conductas, expresiones cotidianas, amalgamadas por un marco normativo común, esta consolidación produce en los individuos un sentido de identidad organizacional, que impulsa el progreso, facilita la convivencia y conduce al respeto de su hábitat laboral o residencial. Es principal objetivo de la cultura ciudadana, generar capital social, ser entendida como la capacidad de interactuar y colaborar entre personas e instituciones, orientada a la obtención del bienestar humano. 
Por cultura cívica o ciudadana, también nos referimos a los comportamientos, actitudes y percepciones de los individuos en torno a diversos temas asociados con su vida diaria, particularmente a su relación con los bienes públicos y con las leyes y normas que les aplican.

La relación entre cultura ciudadana y desarrollo económico no había sido considerada en la mayoría de los modelos económicos ni en las estrategias de los actores de las ciudades, sino hasta años recientes. Entre los promotores de esta nueva visión destacan Putnam y Langes (Citados por IMCO, 2010b), quienes demuestran que siempre la cultura ciudadana ha sido un factor clave en el desarrollo económico de diferentes países, y que aún mantiene vigencia.

Un ciudadano con cultura cívica tiene cuidado sobre los instrumentos y equipos que utiliza, el agua que consume, la basura que genera, los parques y espacios públicos que utiliza, el impacto social que sus conductas o costumbres generan, es respetuoso con sus semejantes, de las leyes y normas ciudadanas, propone, impulsa y ejecuta ideas, es un emprendedor. Ejemplo de la relación ciudadana con las leyes y normas es el cumplimiento de las obligaciones tributarias, el pago de los servicios públicos que usa, el respeto a las normas de tránsito y de convivencia vecinal. Todo esto contribuye a elevar los índices de desarrollo humano.

Según Tarabay y Perinat (2011), un mejor desarrollo humano no puede impulsarse solamente mediante la educación y las inversiones económicas o una combinación entre ambas. Se requiere atender también el problema cultural de las mentalidades, es decir, inducir cambios de conducta 0 comportamientos de agresividad ciudadana, comportamientos que en algunos casos son interpretados como valores positivos e imitados, divulgados 0 alabados por partes o representaciones importantes de la sociedad. 


\section{La Responsabilidad Social Empresarial, Instrumento de Formación de} Ciudadanía.

Según Milian (2015), el concepto de Responsabilidad Social Corporativa o Empresarial (RS) aparece en los Estados Unidos en la década de los 1950, asociado en su origen a la dinámica empresarial, paulatinamente evoluciona y amplía su radio de acción y comprensión social, y hoy se entiende como un concepto fundamental que va indisolublemente unido a la evolución empresarial, rebasa a la simple y elemental filantropía. Así como la empresa cumple con estándares de carácter económico y financiero, para consolidar su praxis empresarial, debe también responsabilizarse de su compromiso legal y moral, independientemente de su tamaño y objeto empresarial, es en este ámbito donde puede inscribirse la acción educativa, consistente en la formación de ciudadanía, la que bajo la figura de la corresponsabilidad le correspondería al sector empresarial.

Diferentes autores e investigadores de la temática, entre ellos Bour (2012), coinciden en atribuir a Bowen la cualidad de precursor o progenitor del término, quien a mediados de los años 1950 opinaba que las acciones de las grandes empresas afectaban la vida de los ciudadanos de diversas maneras y que por tanto era obligación de los empresarios trabajar en función de objetivos y valores sociales, opinión que ya generaba las raíces denominativas del concepto de responsabilidad social de las empresas, que luego es asumido como parte de la filosofía de gestión de múltiples organizaciones y hoy forma parte cotidiana de la dinámica empresarial y organizacional en general, al punto que ya se clasifica en función del sector donde se desenvuelva la organización en cuestión.

En el ordenamiento jurídico venezolano, y específicamente en la Constitución de la República (1999), el concepto tiene un posicionamiento relevante, cuando en su artículo 135, en consonancia con los principios fundamentales de libertad, justicia, igualdad, solidaridad y democracia 
previstos en el titulo primero, establece la norma marco en materia de Responsabilidad Social y dispone que las obligaciones que correspondan al Estado, en cumplimiento de los fines del bienestar social general, no excluyen aquellas que en virtud de la solidaridad y responsabilidad social y asistencia humanitaria, correspondan a los particulares según su capacidad.

\section{Consideraciones Finales}

El mayor reto de toda organización empresarial que pretenda crear cultura ciudadana, será fomentar en cada individuo la aprehensión de una conducta adecuada, entendiéndose que dicha conducta debe ser apropiada por el propio individuo y no ser el resultado de una presión externa. Logrado esto se podría vislumbrar el inicio de una importante evolución ciudadana.

La formación en cultura ciudadana es un aporte individual de quienes conforman una sociedad, tener más y mejores ciudadanos dará lugar a un ambiente más humanitario y saludable. Esta formación, responsabilidad compartida, debe iniciarse en las edades y etapas de la educación más tempranas, y continuada en cada uno de los siguientes niveles, es necesario un acuerdo social de las familias, las instituciones educativas y las organizaciones empresariales, aportando cada una lo necesario a la formación de una mejor ciudadanía.

Definitivamente, no es la cantidad de normas, leyes y demás instrumentos legales, lo que logrará, la creación y consolidación de la cultura ciudadana, esta se corresponde más con actitudes personales que con medios represivos, se corresponde más con convicción que con persuasión, es decir, es en lo más interno del individuo donde debe nacer la comprensión sobre las ventajas de la cultura ciudadana y la convivencia que de ella se deriva.

No es suficiente el concurso de las familias, instituciones educativas, acción docente y organismos públicos. Las organizaciones empresariales en el marco de la corresponsabilidad y la responsabilidad social también están 
llamadas a desarrollar programas en el área de su influencia geográfica o económica, orientados a promover, promocionar, inculcar y fortalecer la cultura ciudadana, sus valores y sus virtudes. Mejores ciudadanos, mejores empleados, mejores empresas, mejor país.

\section{Referencias}

Acosta de Valera, M., Páez, H. y Vizcaya, O. (2007). ¿Es posible educar en valores en las instituciones educativas? Revista Educar en Valores. Año 2007 / Vol. 1 / № 7. Valencia, enero-junio 2007.

Anzola, A. (2012). La corresponsabilidad como principio constitucional en Venezuela. 2012, nro. 26, pp. 3-29. ISSN: 1405-9193. Recuperado de: http://www.scielo.org.mx/scielo.php?script=sci arttext\&pid=S1405$91932012000100001 \& \operatorname{lng}=\mathrm{es} \& \mathrm{nrm}=\mathrm{iso}$

Bour, E. (2012) Responsabilidad social de la empresa análisis del concepto. Revista Estudios Económicos. Vol. XXIX (N.S.), N 59, 1-30. Recuperado de:

https://dialnet.unirioja.es/descarga/articulo/5318733.pdf

Constitución de la República Bolivariana de Venezuela (1999). Gaceta Oficial de la República, № 36.860, del 30 de diciembre.

Fondo Multilateral de Inversiones FOMIN (2011). La responsabilidad social de la empresa en América Latina. Publicación del Banco Interamericano de Desarrollo, mayo 2011. Recuperado de: https://publications.iadb.org/bitstream/handle/11319/5383/La\%20respo nsabilidad\%20social\%20de\%20la\%20empresa\%20en\%20América\%2 OLatina\%20.pdf

Guevara, B. Zambrano de Guerrero y A. Evies, A. (2007). ¿Para qué educar en valores? Revista Educación en Valores. Año 2007 / Vol. 1 / № 7. Institución Laboral. Universidad de Carabobo. Facultad de Ciencias de la Salud. Escuela de Enfermería. Valencia, Venezuela. Recuperado de: 
http://www.ciceana.org.mx/recursos/tribunatura/pdf/Para que educar en valores.pdf

Horrach, J. (2009). Sobre el concepto de ciudadanía: historia y modelos. Revista de Filosofía Factótum 6, pp. 1-22. Recuperado de: https://www.ses.unam.mx/curso2015/pdf/25sep-Horrach.pdf

Instituto Mexicano para la Competitividad IMCO A.C. (2010a,b). Acciones urgentes para las ciudades del futuro. Libro versión digital. Recuperado de:

http://imco.org.mx/ciudades2010/PDFS/Indicedecompetitividadurbana 2010.pdf

Martínez, M. (2010). Educación y Ciudadanía en Sociedades Democráticas: Hacia una ciudadanía colaborativa. Educación, Valores y Ciudadanía. Capitulo IV, pág. 59-71. Publicaciones OIE. Libro versión digital. Recuperado de:

http://www.oei.es/historico/publicaciones/detalle publicacion.php?id=7 Mejías, C. y Henríquez, P. (2012). La Ciudadanía como Co-construcción de Espacios de Participación en lo Público. Revista Sociologías, Porto Alegre, año 14, nro. 31, 2012, pág. 192-213. Sociologías Artigo. Recuperado de: http://www.scielo.br/pdf/soc/v14n31/09.pdf

Milian L. (2015). Origen y Evolución del Concepto de RSC en el entorno Empresarial Europeo y Español. Facultad de Ciencias Económicas y Empresariales. Universidad Pontificia Comillas, Madrid. Recuperado de:

https://repositorio.comillas.edu/xmlui/bitstream/handle/11531/4516/TF G001306.pdf? sequence $=1$

Ministerio de Educación y Deportes de la República Bolivariana de Venezuela (2004). La Educación Bolivariana Políticas, programas y acciones "Cumpliendo las metas del milenio". Caracas. 
Ministerio del Poder Popular para la Planificación y el Desarrollo (2009). Plan de Acción del Programa País Gobierno de la República Bolivariana de Venezuela y EI Fondo de la Naciones Unidas para la Infancia 2009-2013. Dirección General de Cooperación Técnica Internacional y Dirección de Desarrollo Humano y Empleo, República Bolivariana de Venezuela, Fondo de las Naciones Unidas para la Infancia. UNICEF, Venezuela.

Tarabay, F. y Perinat, A. (2011). Educación, cultura y desarrollo humano en Venezuela. Revista Gestión y Gerencia. Volumen 5. Nro. 3. Recuperado de:

http://www.ucla.edu.ve/DAC/investigacion/gyg/GyG\%202011/Diciembr e\%202011/2-\%20FanyTarabay-AdolfoPerinat.pdf 


\section{Elita Marina Méndez Jiménez}

e-mail: elitamendezj@gmail.com; elitamendez@ucla.edu.ve

Nacida en Venezuela. Contador Público (UCLA), Magister

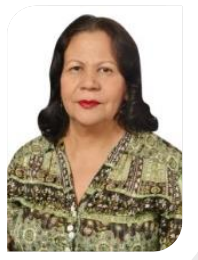
en Finanzas (UBA), Doctora en Gerencia Avanzada (UFT), Doctorado en Finanzas y Contabilidad (DEA- UVA, España) Posdoctorado en Estudios Libres (UFT), acreditada en programas PEII y PEILA. Docente Titular, UCLA, Decanato de Ciencias Económicas y Empresariales, Técnicas Cuantitativas, Cátedra Matemática Financiera. Investigadora en Responsabilidad Social Organizacional, Organizaciones de la Economía Social y Gobernabilidad Universitaria. Tutora y jurado de trabajos de grado y tesis doctorales. Arbitro de revistas científicas. Jurado en concurso premios de investigación. Ponente en eventos científicos. Autora coautora de artículos publicados en revistas Científicas Nacionales. Miembro ASOVAC. 